Research Paper

\title{
Continuation of Tyrosine Kinase Inhibitor is Associated with Survival Benefit in NSCLC Patients with Exon 19 Deletion after Solitary Progression
}

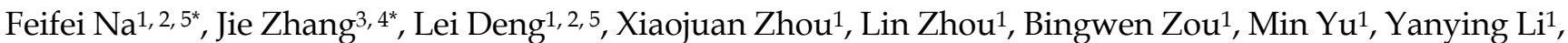
Jianxin Xue ${ }^{1}$, Yongmei Liu ${ }^{1 凶}$

1. Department of Thoracic Cancer, Cancer Center, West China Hospital, West China School of Medicine, Sichuan University, 37 Guoxue Lane, Chengdu, Sichuan, China, 610041;

2. Huaxi Student Society of Oncology Research, West China School of Medicine, Sichuan University, 37 Guoxue Lane, Chengdu, Sichuan, China, 610041;

3. Department of Medical Oncology, The First Affiliated Hospital of Chongqing Medical University, 1 Youyi Rd, Yuzhongqu, Chongqing, China, 400016;

4. Department of Medical Oncology, Cancer Center, West China Hospital, West China School of Medicine, Sichuan University, 37 Guoxue Lane, Chengdu, Sichuan, China, 610041;

5. Department of Thoracic Cancer, Cancer Center and State Key Laboratory of Biotherapy, West China Hospital, West China School of Medicine, Sichuan University and Collaborative Innovation Center, 37 Guoxue Lane, Chengdu, Sichuan, China, 610041.

* These authors contributed equally

$\square$ Corresponding author: Yongmei Liu, MD, Department of Thoracic Cancer, Cancer Center and State Key Laboratory of Biotherapy, West China Hospital, West China School of Medicine, Sichuan University, 37, Guoxue Lane, Chengdu, 610041 Tel: 028-85423571; Fax: 028-85423571; Email: lymi75@163.com

(c) Ivyspring International Publisher. This is an open access article distributed under the terms of the Creative Commons Attribution (CC BY-NC) license (https://creativecommons.org/licenses/by-nc/4.0/). See http://ivyspring.com/terms for full terms and conditions.

Received: 2017.03.10; Accepted: 2017.06.04; Published: 2017.10.12

\begin{abstract}
Introduction: The benefit and selection criteria of continuing tyrosine kinase inhibitor (TKI) after secondary resistance in non-small cell lung cancers (NSCLCs) with epidermal growth factor receptor (EGFR) mutation remain largely unknown. This study was designed to investigate the role and predictive factors of TKI continuation in patients with solitary progression.

Methods: We retrospectively analyzed NSCLCs treated with first generation of TKI from June 2009 to October 2014 in our cancer center. Number of progressive lesions upon first progression was recorded per RECIST v1.1.

Results: Sixty-one of 144 (42.4\%) patients progressed with one lesion. Postprogression TKI use information was available in 58 patients. No brain metastases and stable disease compared to immediate prior scans were associated continued TKI. In the whole cohort, TKI as the first line treatment was found to be associated with longer postprogression survival, but TKI continuation was not. In patients with exon 19 deletion, TKI continuation compared to discontinuation was significantly associated with longer postprogression survival ( 32.0 months, $95 \% \mathrm{Cl}$ : $20.8-43.3$ vs. 15.6 months, $95 \% \mathrm{Cl}: 7.3-23.8, \mathrm{p}=0.013$ ). This difference was not observed in L858R mutation. Exon 19 deletion patients had longer time to TKI cessation after progression (13.7 months, $95 \% \mathrm{Cl}: 4.5-22.9$ vs. 5.6 months in L858R, 95\% Cl: 0.0-11.9, $\mathrm{p}=0.047$ ).

Conclusions: TKI continuation may prolong survival of NSCLCs with exon 19 deletion rather than L858R. Further studies are required to validate this finding.
\end{abstract}

Key words: EGFR mutation; exon 19 deletion; L858R; TKI continuation.

\section{Introduction}

Epidermal growth factor receptor (EGFR) tyrosine kinase inhibitor (TKI) has revolutionized the treatment of non-small cell lung cancer (NSCLC) with EGFR mutation and has become the standard of care in such patients ${ }^{1}$. However, almost all patients eventually develop resistance ${ }^{2}$.
It has been suggested that a subgroup of patients may benefit from TKI continuation despite objective progression, but the criteria of patient selection remain largely unknown. Three clinical resistant modes were proposed based on a cohort of Chinese patients - dramatic, progressive, and local 
progression $^{3}$. Of great interest, longer postprogression survival was found in patients who had received postprogression TKI compared to chemotherapy. A large single arm trial in Asia showed remarkable second progression-free survival (PFS) of 14 months in patients who continued erlotinib after first progression. In this study, choice of erlotinib continuation was not standardized, but was based on decisions of treating physicians, so it remains unclear who would benefit.

T790M mutation is responsible for the majority of resistance ${ }^{4-6}$. It was found to be heterogeneous even among different tumor foci in an individual patient, indicating that some tumors may be still sensitive to first generation of TKI after another population of tumor cells have developed resistance ${ }^{7}$.

However, a recently published randomized phase III trial demonstrated no benefit of continuing gefitinib after objective progression, highlighting the role of correct patient selection in making a decision on continuation or not ${ }^{8}$.

National Comprehensive Cancer Network guideline recently included continuation of first generation of TKI after solitary progression in conjunction with local therapy as one of the treatment options if symptomatic ${ }^{9}$. In this study, we aimed to investigate the benefit of continuing first generation of TKI in patients with solitary progression and to explore factors that may assist patient selection.

\section{Material and Methods}

The Institutional Review Board of West China Hospital reviewed and approved this study. Written informed consent was obtained from all participants involved in the study. Medical records of TKI-naïve metastatic NSCLC patients with common EGFR mutation (exon 19 deletion and L858R) treated with first generation of EGFR-TKIs between June 2009 to October 2012 were retrospectively reviewed. Imaging workup at baseline typically involved chest and abdominal computed tomography (CT) and brain magnetic resonance imaging (MRI). Patients were followed typically every two months with routine chest and abdominal CT. Follow-up brain MRI was performed if clinically indicated. We reviewed images according to RECIST 1.1 and recorded the number of progressive lesions ${ }^{10}$. EGFR mutation was determined by ADx-ARMS EGFR Mutations Detection Kit (Amoy Diagnostics, Xiamen, China).

PFS was defined from first TKI initiation till objective progression and/or death. Longer PFS was defined as a PFS longer than the median PFS of patients with solitary progression. Change of scanning at the time of progression compared to immediate preceding scan was recorded as stable disease (SD) or progressive disease (PD) per RECIST 1.1 and was considered a surrogate clinical index of tumor growth rate. The followings were considered as definitive local therapy after progression: 1) surgical resection; 2) conventional fractionated radiotherapy with a total dose $>=50 \mathrm{~Gy}$; 3) stereotactic body radiotherapy with a biological equivalent dose $>=100$ Gy; and 4) stereotactic radiosurgery to brain metastases. Solitary progression was defined as only a single lesion progress and the rest of lesions were stable in patients with multisite lesions.

Three first generation EGFR-TKIs have been approved in China: gefitinib, erlotinib, and icotinib. Patients who switched to another TKI after objective progression were still considered as TKI continuation. TKI continuation was defined as no cessation of TKI use for more than one month after progression. Age, gender, smoking history, line of TKI, ECOG performance status, new/original site progression, presence of brain metastasis, and chemotherapy use were also collected. Chemotherapy given within 2 month postprogression was considered as immediate.

Postprogression survival was defined as from first objective TKI progression till any causes of death. Chi-square or Fisher's exact test was used to analyze factors associated with TKI continuation. Survival was estimated by Kaplan-Meier method and compared by logrank test. Multivariate analyses of factors associated with postprogression survival was performed by Cox regression test with forward conditional method. All statistical analyses were performed by IBM SPSS Statistics v22.

\section{Results}

61 of $144(42.4 \%)$ patients progressed with one lesion after a median PFS of 11.9 months (95\% CI: 9.9 -13.9). Median postprogression survival was 21.1 months (95\% CI: 12.2 - 30.1). Postprogression TKI use information was available in 58 of the 61 patients. Median time of follow-up from progression was 14.4 months (range, 0.6 - 59.8).

\section{Patient characteristics and association with TKI continuation}

Patient characteristics were summarized in Table 1. Demographical distribution of smoking history and histology is consistent with the previous reports of patients with EGFR mutation. EGFR-TKI was the first line of systemic treatment in $43(74.1 \%)$ patients. 41 $(70.7 \%)$ patients progressed in an original site and 39 $(67.2 \%)$ had stable disease compared to preceding scan. $12(20.7 \%)$ patients received definitive local therapy either to primary or metastatic lesions after progression, but none received both. All definitive metastatic local therapies were stereotactic 
radiosurgery to brain lesions. 5 of 32 patients with exon 19 deletion received definitive local therapy; while 6 of 26 L858R received. No patient received radiofrequency ablation after progression. One patient received AZD9291, but no other patients received third generation of TKI.

Table 1. Patient Characteristics Stratified by Tyrosine Kinase Inhibitor (TKI) Continuation

\begin{tabular}{|c|c|c|c|c|c|}
\hline & \multicolumn{2}{|c|}{$\begin{array}{l}\text { TKI } \\
\text { Discontinuation }\end{array}$} & \multicolumn{2}{|c|}{$\begin{array}{l}\text { TKI } \\
\text { Continuation }\end{array}$} & P value \\
\hline & Number & $\%$ & \multicolumn{2}{|c|}{ Number \% } & \\
\hline Total & 21 & & 37 & & \\
\hline \multicolumn{5}{|l|}{ Age (y) } & 0.729 \\
\hline$<65$ & 18 & $85.7 \%$ & 29 & $78.4 \%$ & \\
\hline \multicolumn{5}{|l|}{ Sex } & 0.702 \\
\hline Female & 13 & $61.9 \%$ & 21 & $56.8 \%$ & \\
\hline \multicolumn{5}{|l|}{ Smoker } & 0.741 \\
\hline Non-smoker & 16 & $76.2 \%$ & 30 & $81.1 \%$ & \\
\hline \multicolumn{6}{|l|}{ ECOG performance status } \\
\hline $0-1$ & 21 & $100.0 \%$ & 37 & $100.0 \%$ & \\
\hline \multicolumn{5}{|l|}{ Histology } & 0.743 \\
\hline Adenocarcinoma & 20 & $95.2 \%$ & 34 & $91.9 \%$ & \\
\hline Squamous carcinoma & 1 & $4.8 \%$ & 2 & $5.4 \%$ & \\
\hline Adenosquamous carcinoma & 0 & $0.0 \%$ & 1 & $2.7 \%$ & \\
\hline \multicolumn{5}{|l|}{ Mutation Type } & 0.437 \\
\hline Exon 19 deletion & 13 & $61.9 \%$ & 19 & $51.4 \%$ & \\
\hline L858R & 8 & $38.1 \%$ & 18 & $48.6 \%$ & \\
\hline \multicolumn{5}{|l|}{ Line of TKI } & 0.788 \\
\hline 1 & 16 & $76.2 \%$ & 27 & $73.0 \%$ & \\
\hline$>=2$ & 5 & $23.8 \%$ & 10 & $27.0 \%$ & \\
\hline \multicolumn{5}{|l|}{ Brain metastases } & $<0.001$ \\
\hline Yes & 19 & $90.5 \%$ & 5 & $13.5 \%$ & \\
\hline \multicolumn{5}{|c|}{ Number of Extracranial Lesions } & 1.000 \\
\hline$<=6$ & 17 & $81.0 \%$ & 30 & $81.1 \%$ & \\
\hline \multicolumn{5}{|c|}{ Scan comparison with immediate prior scan } & 0.016 \\
\hline Progressive Disease & 11 & $52.4 \%$ & 8 & $21.6 \%$ & \\
\hline Stable Disease & 10 & $47.6 \%$ & 29 & $78.4 \%$ & \\
\hline \multicolumn{5}{|l|}{ Progressive site } & 0.268 \\
\hline Original Site & 13 & $61.9 \%$ & 28 & $75.7 \%$ & \\
\hline New Site & 8 & $38.1 \%$ & 9 & $24.3 \%$ & \\
\hline \multicolumn{5}{|c|}{ Prior progression-free survival (months) } & 0.585 \\
\hline Long & 9 & $42.9 \%$ & 20 & $54.1 \%$ & \\
\hline Short & 12 & $57.1 \%$ & 17 & $45.9 \%$ & \\
\hline \multicolumn{6}{|l|}{ Treatment after progression } \\
\hline \multicolumn{5}{|l|}{ Immediate Chemotherapy } & 0.040 \\
\hline Yes & 10 & $47.6 \%$ & 8 & $21.6 \%$ & \\
\hline \multicolumn{5}{|c|}{ Chemotherapy at any time postprogression } & 0.760 \\
\hline Yes & 15 & $71.4 \%$ & 25 & $67.6 \%$ & \\
\hline \multicolumn{5}{|c|}{ Definitive primary local therapy } & 0.402 \\
\hline Yes & 1 & $4.8 \%$ & 5 & $13.5 \%$ & \\
\hline \multicolumn{5}{|l|}{ Definitive metastatic local therapy } & 0.148 \\
\hline Yes & 0 & $0.0 \%$ & 5 & $13.5 \%$ & \\
\hline \multicolumn{5}{|c|}{ Definitive local therapy to any site } & 0.036 \\
\hline Yes & 1 & $4.8 \%$ & 10 & $27.0 \%$ & \\
\hline
\end{tabular}

Patient treatment characteristics stratified by TKI Continuation were summarized in Table 4. 15 $(71.43 \%)$ patients in TKI continuation group received chemotherapy, and $12(57.14 \%)$ patients received platinum-based regimen (doublet) treatment; in TKI continuation group, 25(67.57\%) patients received chemotherapy including $15(40.54 \%)$ platinum-based regimen (doublet) and 10(27.03\%) single-drug cytotoxic. Use of SBRT or CRT radiotherapy after postprogression of the study radiotherapy was greater in TKI continuation group than in TKI discontinuation group. In TKI continuation group $2(5.4 \%)$ patients received SBRT, the strategy was 50Gy/10f and 40Gy/8f respectively; 8 (21.62\%) patients underwent CRT which total dosage ranged from 30Gy to 66Gy, and all the radiotherapy were confined to the area of the solitary progression lesion. In TKI discontinuation group, there was only one patient received CRT with 40Gy total dose.

$37(63.8 \%)$ patients continued EGFR-TKI after objective progression. No significant difference of prior PFS was observed in patients who continued TKI (11.7 months, 95\% CI: 8.6 - 14.8) versus those who discontinued (12.7 months, 95\% CI: 7.4 - 18.1). Patients without brain metastases $(94.1 \%$ continued vs. $20.8 \%$ in those with, $\mathrm{p}<0.001$ ) and stable disease compared to immediate prior scan $(74.4 \%$ continued vs. $42.1 \%$ in those with PD, $\mathrm{p}=0.016$ ) were significantly associated with TKI continuation. Patients who continued TKI $(27.0 \%$ vs. $4.8 \%$ in those discontinued) were more likely to have receive definitive local therapy to either primary or metastatic site $(\mathrm{p}=0.036)$ after progression. Immediate chemotherapy was more common in TKI discontinuation group ( $47.6 \%$ vs. $21.6 \%, \mathrm{p}=0.040)$.

\section{Postprogression survival in the whole cohort}

Patients who continued TKI did not have a significantly longer postprogression survival $(p=0.28$, Figure 1A). On multivariate analyses, only TKI used in the first line setting (hazard ratio: $0.43,95 \% \mathrm{CI}: 0.16$ - 0.83) was significantly associated with longer postprogression survival (Table 2). In subgroup analysis, exon 19 deletion (hazard ratio: $0.31,95 \% \mathrm{CI}$ : 0.06-0.67) and long prior PFS (hazard ratio: 0.34, 95\% CI: 0.46-0.82) significantly favored TKI continuation (Figure 2).

\section{Postprogression survival stratified by mutation type}

In patients with exon 19 deletion mutation, TKI continuation was associated with significantly longer postprogression survival (32.0 months, 95\% CI: 20.8 43.3 vs. 15.6 months, 95\% CI: 7.3 - 23.8, $\mathrm{p}=0.013$ ). Among other factors, line of TKI was also significant (Table 3). On multivariate analyses, only TKI continuation remained significant $(p=0.023)$. However, in patients with L858R mutation, no benefit of continuing TKI was observed. SD compared to immediate prior scan, original site failure and no brain metastases were associated with longer postprogression survival on multivariate analyses in L858R mutation patients. 


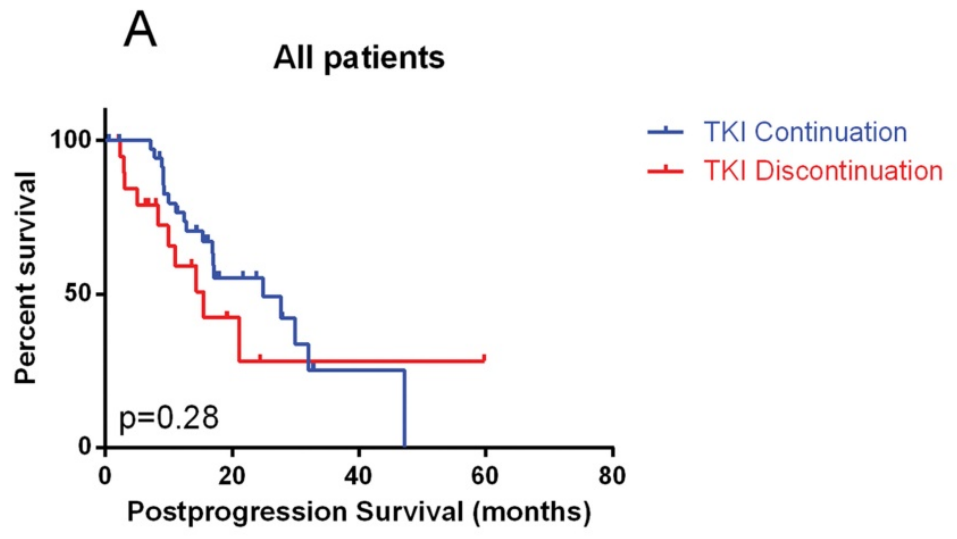

B

Exon 19 deletion

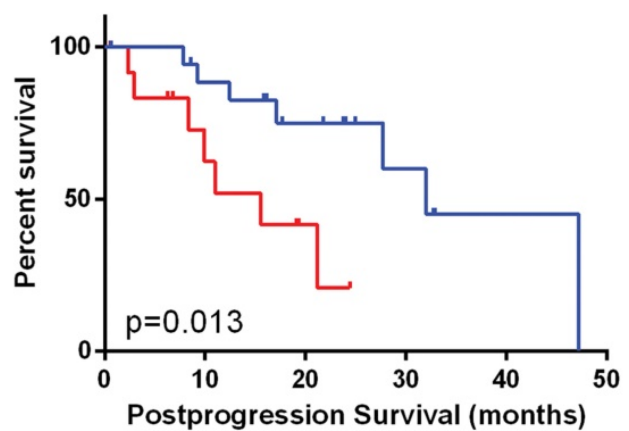

L858R

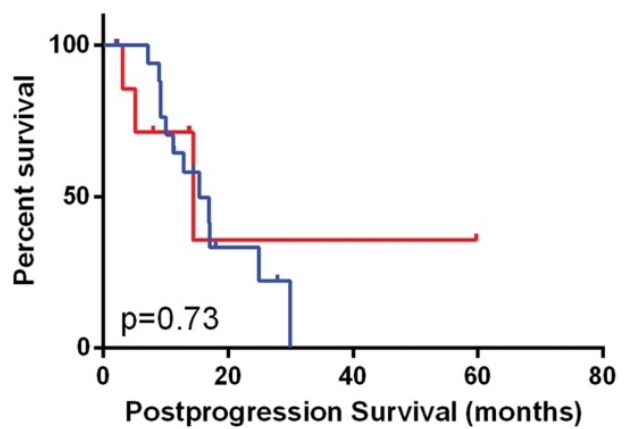

Figure 1. Postprogression survival in A) whole cohort; B) exon 19 deletion mutation; C) L858R mutation

In patients who continued TKI, exon 19 deletion was significantly associated with longer postprogression time to TKI cessation (13.7 months, 95\% CI: $4.5-22.9$ vs. 5.6 months in L858R, 95\% CI: $0.0-11.9, \mathrm{p}=0.047$, Figure 3).

\section{Discussion}

This study showed that in unselected EGFR mutant patients with solitary progression after TKI treatment, TKI continuation was not associated with longer postprogression survival. Interestingly, subgroup analysis showed that patients with exon 19 deletion rather than L858R mutation may benefit from TKI continuation, evidenced by longer postprogression survival and longer time to TKI cessation. To the best of our knowledge, this is the first study investigating difference of postprogression TKI continuation between the two common EGFR mutation in patients with solitary progression.

Although both are common, exon 19 deletion and L858R have been considered to be different in its biology and treatment response to TKI. A pooled analysis of LUNX-LUNG 3 and 6 demonstrated overall survival benefit of afatinib, an EGFR-TKI in exon 19 deletion patients, but not in L858R over chemotherapy ${ }^{11}$. A meta-analysis of 7 randomized trials also found $50 \%$ greater PFS benefit of exon 19 deletion compared to patients with L858R. This efficacy difference may be explained by a greater degree of EGFR phosphorylation inhibition by gefitinib in a preclinical study ${ }^{12}$. However, whether this difference exists in patients who continues TKI after progression has been largely unknown.

Table 2. Factors associated with postprogression survival

\begin{tabular}{lll}
\hline & $\begin{array}{l}\text { Univariate P } \\
\text { value }\end{array}$ & $\begin{array}{l}\text { Multivariate P } \\
\text { value }\end{array}$ \\
\hline Age $>=65$ (year) & 0.027 & 0.117 \\
Gender & 0.255 & \\
Smoking & 0.911 & \\
Line of tyrosine kinase inhibitor & 0.018 & 0.022 \\
Tumor growth rate (PD or SD) & 0.474 & \\
Mutation type & 0.165 & \\
Number of extracranial lesions & 0.307 & \\
Brain metastases & 0.654 & \\
Immediate chemotherapy & 0.156 & \\
Progressive site & 0.023 & \\
Definitive local therapy to metastatic & 0.111 & \\
site(s) & & \\
Definitive local therapy to primary & 0.343 & \\
site(s) & & \\
Definitive local therapy to any site(s) & 0.053 & \\
Continuation of tyrosine kinase inhibitor & 0.283 & \\
Longer progression-free survival & 0.602 & \\
\hline PD: progressive disease; SD: stable disease & &
\end{tabular}




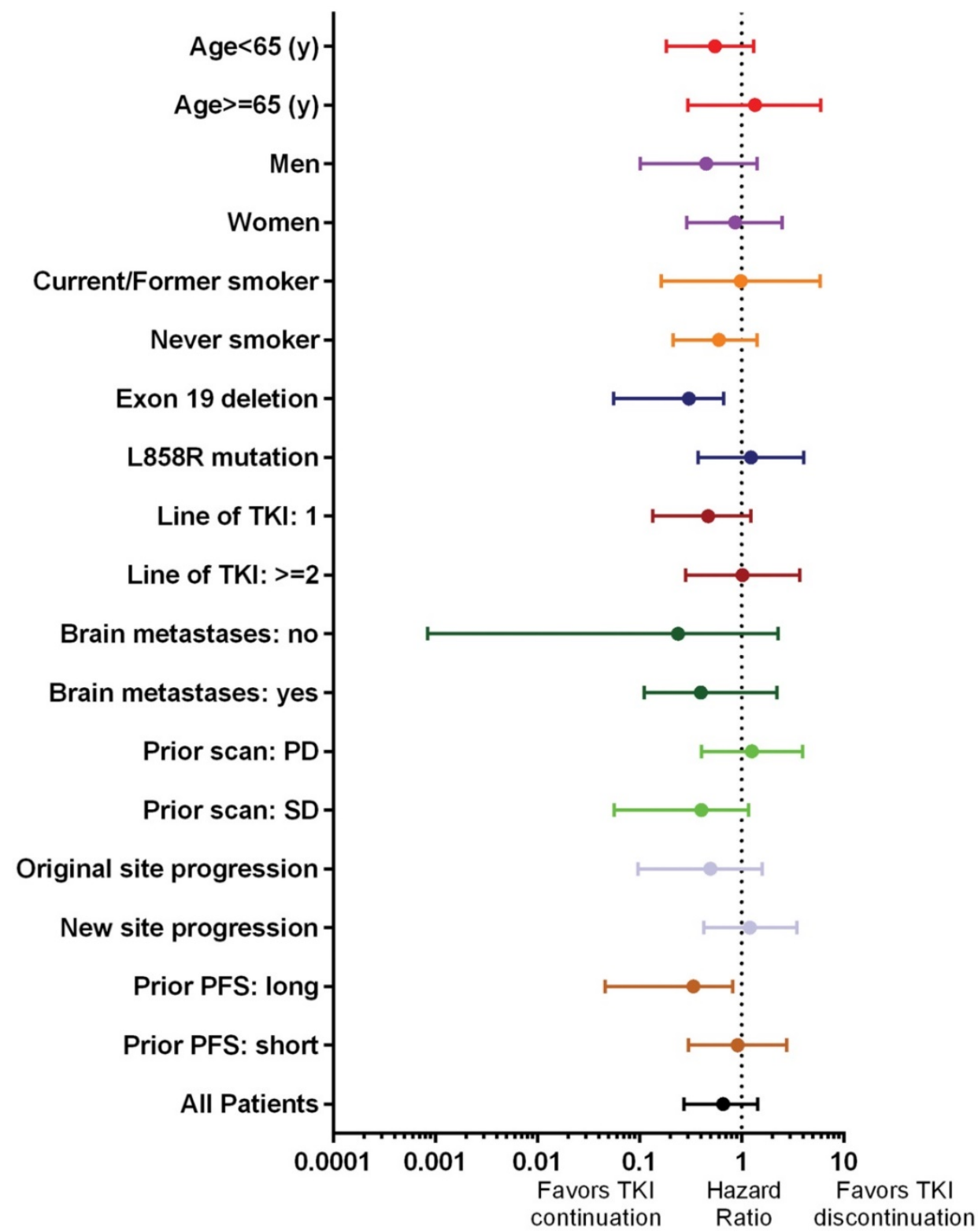

Figure 2. Forest plot of postprogression survival subgroup analyses. Only 11 patients received definitive local therapy and analysis was not possible. TKI: tyrosine kinase inhibitor; PD: progressive disease; SD: stable disease; PFS: progression-free survival

Table 3. Factors associated with postprogression survival stratified by mutation type

\begin{tabular}{|c|c|c|c|c|}
\hline & \multicolumn{2}{|l|}{ Exon 19 deletion } & \multicolumn{2}{|l|}{ L858R } \\
\hline & Univariate $\mathrm{p}$ value & Multivariate $\mathrm{p}$ value & Univariate $\mathrm{p}$ value & Multivariate $p$ value \\
\hline Age $(>=65 y)$ & 0.824 & & 0.200 & \\
\hline Female & 0.743 & & 0.214 & \\
\hline Non-smoker & 0.673 & & 0.310 & \\
\hline Line of tyrosine kinase inhibitor & 0.038 & 0.081 & 0.235 & \\
\hline Slow tumor growth (stable disease) & 0.300 & & 0.876 & 0.007 \\
\hline Original site progression & 0.681 & & 0.006 & $<0.001$ \\
\hline Number of extracranial lesions & 0.812 & & 0.300 & \\
\hline Brain metastases & 0.111 & & 0.641 & 0.032 \\
\hline Immediate chemotherapy & 0.476 & & 0.559 & \\
\hline Any definitive local therapy & 0.166 & & 0.110 & \\
\hline Tyrosine kinase inhibitor continuation & 0.013 & 0.023 & 0.733 & \\
\hline Longer progression-free survival & 0.720 & & 0.336 & \\
\hline
\end{tabular}


Table 4. Treatment Characteristics Stratified by Tyrosine Kinase Inhibitor (TKI) Continuation

\begin{tabular}{|c|c|c|c|c|}
\hline & \multicolumn{2}{|c|}{ TKI Discontinuation } & \multicolumn{2}{|c|}{ TKI Continuation } \\
\hline & Number & $\%$ & Number & $\%$ \\
\hline \multicolumn{5}{|c|}{ Patient who received chemotherapy } \\
\hline Platinum alone* & 0 & 0 & 1 & $2.70 \%$ \\
\hline $\begin{array}{l}\text { Platinum-based regimen } \\
\text { (doublets) }\end{array}$ & 12 & $57.14 \%$ & 15 & $40.54 \%$ \\
\hline Other single-drug*** & 3 & $14.29 \%$ & 10 & $27.03 \%$ \\
\hline \multicolumn{5}{|c|}{ Patient who received radiotherapy } \\
\hline SBRT & 0 & 0 & 2 & $5.40 \%$ \\
\hline CRT & 1 & $4.70 \%$ & 8 & $21.62 \%$ \\
\hline
\end{tabular}

${ }^{*}$ Carboplatin, cisplatin, or nedaplatin.

**Docetaxel, paclitaxel, pemetrexed, gemcitabine, or vinorelbine.

SBRT: Stereotactic Body Radiation Therapy CRT: Conventional Radiation Therapy

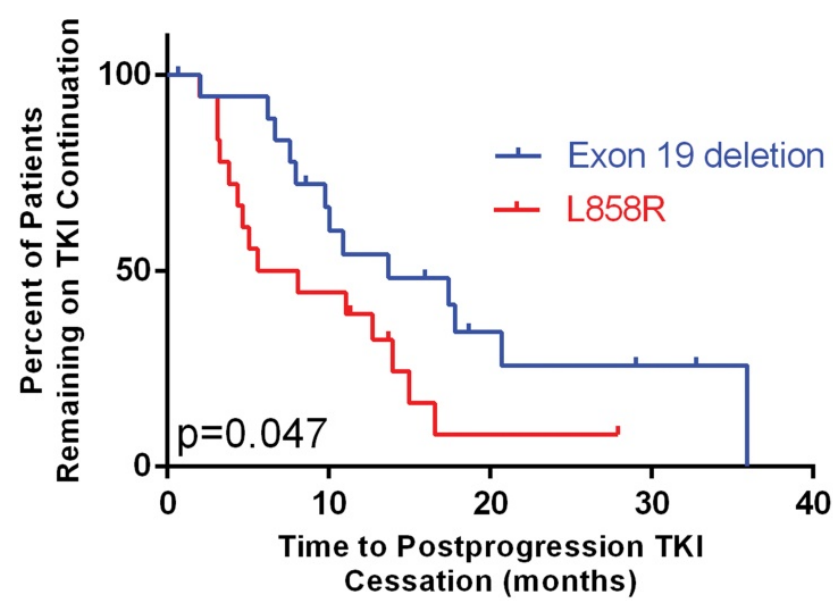

Figure 3. Time to tyrosine kinase inhibitor (TKI) cessation after progression stratified by mutation type

The benefit of TKI continuation after progression has not been confirmed. Neither consensus on patient selection criteria has been reached. The IMPRESS study demonstrated no benefit of gefitinib continuation in unselected patients ${ }^{8}$. The post-resistance PFS was 5.4 months, identical between patients with or without gefitinib continuation. Interestingly, its subgroup analysis showed a trend toward a longer second PFS in patients with exon 19 deletion over L858R mutation, but overall survival result has not been reported. The ASPIRATION study, a single-arm phase II trial showed that in patients selected by treating physicians, the postprogression PFS by erlotinib continuation was 14.1 months, which was even numerically longer than the first PFS (11.0 months) ${ }^{13}$. The discrepancy between IMPRESS and ASPIRATION studies suggests that with the right selection, TKI may still be beneficial in a subgroup of patients. However, the selection criteria remain largely unknown. Subgroup analysis of this study showed that patients with exon 19 deletion and long prior PFS benefited from TKI continuation
(Figure 2). Because of the objective nature of mutation type, we therefore chose exon 19 deletion for further analysis.

Salvage local therapy has been used increasingly in selected asymptomatic patients with limited number of progressive lesions, good performance status, and small number of total metastatic burdens. A retrospective analysis from a single institution showed that oligometastatic and solitary progressive EGFR mutant patients achieved an impressive 10 months of median time to progression after local therapy ${ }^{14}$. They also reported the median time until a subsequent change in systemic therapy was 22 months. The benefit of local therapy has been reported by other studies as well ${ }^{15}, 16$. The latest NCCN guideline included local therapy beyond focal progression as a treatment option ${ }^{9}$. However, it is hard to determine the benefit of local therapy in this cohort of patients, because of the small number of patients who received definitive local therapy.

With the advent of third generation of TKI, continuation of first generation of TKI appears to be less appealing ${ }^{17-19}$. However, new resistances to those novel drugs have emerged after a certain time of treatment as in the first generation drugs ${ }^{20,21}$. On the other hand, the mechanism of TKI resistance is complex, except the presence of new mutations in EGFR gene, such as T790M and C797520, PI3K mutation or MET amplification and pathological transformation also play an important role in acquired resistance to EGFR-TKI22, 23. Therefore, continuation of first generation of TKI seems to be reasonable as long as patient can still benefit, considering the cost and inevitable subsequent resistance from the newer drugs.

This study has the typical limitations of retrospective studies and is small in patient numbers. The postprogression treatment is also heterogeneous, including concurrent chemotherapy + TKI, TKI alone, chemotherapy alone, etc. Therefore, we grouped patients into TKI continuation and discontinuation and used postprogression survival as the outcome endpoint instead of postprogression PFS. Besides, as the patient's characteristics between the TKI discontinuation group and TKI continuation group were not balanced, so the interpretation of the postprogression survival subgroup analyses forest plot needs to be more careful.

In conclusion, this study showed that in EGFR-mutant patients with solitary progression, EGFR TKI continuation compared to discontinuation was associated with longer postprogression survival in exon 19 deletion patients, but not in L858R and unselected patients. Time to TKI cessation was also significantly longer in patients with exon 19 deletion. 
This result should be validated in larger and prospective studies.

\section{Acknowledgement}

This work was supported by National Natural Science Foundation of China [grant numbers: 81602670]; and National Natural Science Foundation of China [grant number: 81602008]. The funding agencies only provide financial support and are not involved in any other parts of this study. https://isisn.nsfc.gov.cn/egrantindex/funcindex/prj search-list

\section{Ethical approval}

All procedures performed in studies involving human participants were in accordance with the ethical standards of the institutional and/or national research committee and with the 1964 Helsinki declaration and its later amendments or comparable ethical standards. For this type of study formal consent is not required.

\section{Competing Interests}

The authors have declared that no competing interest exists.

\section{References}

1. Mok TS, Wu YL, Thongprasert S, et al. Gefitinib or carboplatin-paclitaxel in pulmonary adenocarcinoma. The New England journal of medicine 2009;361:947-957.

2. Zhou C Yao LD Strategies to Improve Outcomes of Patients with EGRF-Mutant Non-Small Cell Lung Cancer: Review of the Literature. Journal of thoracic oncology : official publication of the International Association for the Study of Lung Cancer 2016;11:174-186.

3. Yang JJ, Chen HJ, Yan $\mathrm{HH}$, et al. Clinical modes of EGFR tyrosine kinase inhibitor failure and subsequent management in advanced non-small cell lung cancer. Lung cancer (Amsterdam, Netherlands) 2013;79:33-39.

4. Wheeler DL, Dunn EF, Harari PM. Understanding resistance to EGFR inhibitors-impact on future treatment strategies. Nature reviews Clinical oncology 2010;7:493-507.

5. Gazdar AF. Activating and resistance mutations of EGFR in non-small-cell lung cancer: role in clinical response to EGFR tyrosine kinase inhibitors. Oncogene 2009;28 Suppl 1:S24-31.

6. Yu HA, Arcila ME, Rekhtman N, et al. Analysis of tumor specimens at the time of acquired resistance to EGFR-TKI therapy in 155 patients with EGFR-mutant lung cancers. Clinical cancer research : an official journal of the American Association for Cancer Research 2013;19:2240-2247.

7. Piotrowska Z, Niederst MJ, Karlovich CA, et al. Heterogeneity Underlies the Emergence of EGFRT790 Wild-Type Clones Following Treatment of T790M-Positive Cancers with a Third-Generation EGFR Inhibitor. Cancer discovery 2015;5:713-722

8. Soria JC, Wu YL, Nakagawa K, et al. Gefitinib plus chemotherapy versus placebo plus chemotherapy in EGFR-mutation-positive non-small-cell lung cancer after progression on first-line gefitinib (IMPRESS): a phase 3 randomised trial. The Lancet Oncology 2015;16:990-998.

9. Network NCC. Non-small Cell Lung Cancer (Version 4.2016). Available at https://www.nccn.org/professionals/physician_gls/pdf/nscl.pdf.

10. Eisenhauer EA, Therasse P, Bogaerts J, et al. New response evaluation criteria in solid tumours: revised RECIST guideline (version 1.1). European journal of cancer 2009;45:228-247.

11. Yang JC, $\mathrm{Wu} \mathrm{YL}$, Schuler $\mathrm{M}$, et al. Afatinib versus cisplatin-based chemotherapy for EGFR mutation-positive lung adenocarcinoma (LUX-Lung 3 and LUX-Lung 6): analysis of overall survival data from two randomised, phase 3 trials. The Lancet Oncology 2015;16:141-151.

12. Zhu JQ Zhong WZ, Zhang GC, et al. Better survival with EGFR exon 19 than exon 21 mutations in gefitinib-treated non-small cell lung cancer patients is due to differential inhibition of downstream signals. Cancer letters 2008;265:307-317.

13. Park K, Yu CJ, Kim SW, et al. First-Line Erlotinib Therapy Until and Beyond Response Evaluation Criteria in Solid Tumors Progression in Asian Patients
With Epidermal Growth Factor Receptor Mutation-Positive Non-Small-Cell Lung Cancer: The ASPIRATION Study. JAMA oncology 2016;2:305-312.

14. Yu HA, Sima CS, Huang J, et al. Local therapy with continued EGFR tyrosine kinase inhibitor therapy as a treatment strategy in EGFR-mutant advanced lung cancers that have developed acquired resistance to EGFR tyrosine kinase inhibitors. Journal of thoracic oncology : official publication of the International Association for the Study of Lung Cancer 2013;8:346-351.

15. Conforti F, Catania C, Toffalorio F, et al. EGFR tyrosine kinase inhibitors beyond focal progression obtain a prolonged disease control in patients with advanced adenocarcinoma of the lung. Lung cancer (Amsterdam, Netherlands) 2013;81:440-444

16. Weickhardt AJ, Scheier B, Burke JM, et al. Local ablative therapy of oligoprogressive disease prolongs disease control by tyrosine kinase inhibitors in oncogene-addicted non-small-cell lung cancer. Journal of thoracic oncology : official publication of the International Association for the Study of Lung Cancer 2012;7:1807-1814.

17. Sequist LV, Soria JC, Goldman JW, et al. Rociletinib in EGFR-mutated non-small-cell lung cancer. The New England journal of medicine 2015;372:1700-1709.

18. Janne PA, Yang JC, Kim DW, et al. AZD9291 in EGFR inhibitor-resistant non-small-cell lung cancer. The New England journal of medicine 2015;372:1689-1699.

19. Yver A. Osimertinib (AZD9291)-a science-driven, collaborative approach to rapid drug design and development. Annals of oncology : official journal of the European Society for Medical Oncology / ESMO 2016;27:1165-1170.

20. Thress KS, Paweletz CP, Felip E, et al. Acquired EGFR C797S mutation mediates resistance to AZD9291 in non-small cell lung cancer harboring EGFR T790M. Nature medicine 2015;21:560-562.

21. Planchard D, Loriot Y, Andre F, et al. EGFR-independent mechanisms of acquired resistance to AZD9291 in EGFR T790M-positive NSCLC patients. Annals of oncology : official journal of the European Society for Medical Oncology / ESMO 2015;26:2073-2078.

22. Engelman JA, Zejnullahu K, Mitsudomi T, et al. MET amplifi cation leads to gefi tinib resistance in lung cancer by ctivating ERBB3 signaling. Science 2007; 316: 1039-43.

23. Sequist LV, Waltman BA, Dias-Santagata D, et al. Genotypic and histological evolution of lung cancers acquiring resistance to EGFR inhibitors. Sci Transl Med 2011; 3: 75ra26. 